\title{
A case of Clostridium perfringens sepsis in the 29 week pregnant woman with diagnosed fetal omphalosel
}

\author{
Nezahat Gurler ${ }^{1}$, Lutfiye Oksuz ${ }^{1}$, Bahar Yuksel ${ }^{2}$, Recep Has ${ }^{2}$ \\ ${ }^{1}$ Istanbul Faculty of Medicine, Department of Medical Microbiology, Istanbul-Turkey \\ ${ }^{2}$ Istanbul Faculty of Medicine, Department of Gynecology, Istanbul-Turkey
}

\section{Email address:}

ngurler@istanbul.edu.tr (N. Gurler), loksuz34@yahoo.com (L. Oksuz)

\section{To cite this article:}

Nezahat Gurler, Lutfiye Oksuz, Bahar Yuksel, Recep Has. A Case of Clostridium Perfringens Sepsis in the 29 Week Pregnant Woman with Diagnosed Fetal Omphalosel, Clinical Medicine Research. Vol. 2, No. 3, 2013, pp. 29-31. doi: 10.11648/j.cmr.20130203.12

\begin{abstract}
Although not common, postpartum and post-abortion infections caused by clostridia can be severe. Clostridial uterine infections start as localized chorioamnionitis as a result of an infection from the fetus and/or placental tissues. The infection may spread to the uterine wall and endometrial tissues, and, in the most severe cases, uterine necrosis accompanied by sepsis ensues. In this paper we report the presence of Clostridium perfringens isolated from blood culture and placental specimen after the termination of a 29 gestational week pregnancy. The woman had a history of having omphaloceled fetus and premature membrane rupture. Fetal omphalocele is a congenital defect of the abdominal wall that allows some of the abdominal organs to protrude through it. In order to find the focus of infection, blood and plasental specimen of the patient were examined. Blood cultures of the patient were evaluated by the BACTEC 9120 system. The identification of the bacteria was performed conventionally and confirmed by the automatized API 20A system. Antibiotic susceptibility testing was performed by E-test according to the recommendations of Clinical Laboratory and Standards Institute (CLSI). Although Clostridium spp is rarely isolated from blood cultures, anaerobically evaluation of blood cultures in the infections of abdominal and genital sites could be life-saving, as in this report.
\end{abstract}

Keywords: Clostridium Perfringens, Blood Culture, Placental Specimen, Fetal Omphalocel

\section{Introduction}

Clostridia can be isolated from the genital tract of approximately $10 \%$ of women as part of the normal vaginal microflora. Clostridia are present in up to $20 \%$ of non-sexually transmitted diseases (STD) and may be present as part of bacterial vaginosis. Although not common, postpartum and post-abortion infections caused by clostridia can be severe. Clostridial uterine infections start as localized chorioamnionitis as a result of infection of the fetus and placental tissues. The infection may spread to the uterine wall and endometrial tissues, and, in the most severe cases, uterine necrosis accompanied by sepsis ensues ${ }^{(1)}$.

Although obligate anaerobes are infrequently isolated from bacteremic patients, Clostridia are second only to Bacteroides among anaerobes isolated from blood cultures, accounting for approximately $1 \%$ of all positive cultures. Patients undergoing treatments that render them neutropenic are also at increased risk for clostridial bacteremia. C. perfringens followed by $C$. septicum is the most common species isolated from blood. If not treated promptly the toxins produced by clostridia during septicemia can result in severe disease and devastating clinical outcomes ${ }^{(1)}$. It's reported that $C$. perfringens was the most frequently isolated microorganism in clostridial septicemias, but $C$. septicum was associated with more complications and a higher mortality rate $^{(7)}$.

Fetal omphalocele is a congenital defect of the abdominal wall that allows some of the abdominal organs to protrude through it. A translucent membrane or sac covers the protruding organs. The severity of the condition varies from child to child. In a normal baby, the intestines grow and develop outside the abdomen in the umbilical cord until about the tenth week of pregnancy. After this time the intestines return into the abdomen; when this fails to happen, a fetal omphalocele occurs ${ }^{(9)}$. Omphalocele is a defect of the abdominal wall affecting 1 in 4000 to 7000 live births. Abnormal development of the abdominal muscles, fascia and skin leads to the herniation of abdominal viscera in a sac consisting of amnion and peritoneum. The embryologic process accounting for omphalocele is unclear ${ }^{(5,9) \text {. }}$

In this paper we report $C$. perfringens isolated from blood 
culture and placental specimen after the termination of a 29 gestational week pregnancy.

\section{Case}

A 34 years old, 29 gestational week pregnant women was admitted to emergency services with abdominal pain and fever. The woman had a history of having omphaloceled (but with neither any diagnostic tests like amniocentesis nor cordocentesis was performed) fetus and with a history of premature membrane rupture for a five days. In physical examination abdominal tenderness, foul-smelling vaginal discharge with $38.9^{\circ} \mathrm{C}$ fever and tachycardia (118 beats $\left./ \mathrm{min}\right)$ was diagnosed. Obstetrical ultrasonography showed an omphaloseled mort fetus. In complete blood count white blood cells (WBC) were 20.000 cells $/ \mathrm{ml}$ and C-reactive protein (CRP) level was 95. Blood and urine cultures were made. Cesarean section was performed because of pre-diagnosis of chorioamnionitis. The fetus was born dead with a non-ruptured omphalocele sac. Preoperative blood, urine samples and sample of placenta were sent to the microbiology laboratory. Postoperatively, due to the declining blood parameters (hematocrites -HTC- level decreased from 36 to 24 , international normalised ratio -INR- level increased from 1.00 to 2.1 lactate dehydrogenase -LDH- level also rised up to 900) of the patient was admitted to intensive care unit with a prediagnosis of disseminated intravascular coagulation (DIC) caused by sepsis. The patient was treated with imipenem and doxycycline. Urine culture of the patient was sterile. C. perfringens was isolated from blood culture and placental samples of the patient who had neither an immune deficiency nor a previously diagnosed disease. The isolated microorganism was sensitive to penicillins. On the seventh day of therapy, inflamatory parameters of the patient decreased. And patient was discharged on the postoperatively fifteenth day with normalized complete blood count parameters and inflamation parameters (WBC: 7800 cells/ ml, HTC: 30.6, CRP: 4.7 and INR: 1.2$)$

\section{Microbiological Methods}

The placental sample obtained from the patient was inoculated onto sheep-blood agar, Schaedler agar, Schaedler broth and Gram stain was performed. Gram positive rods were seen in Gram stain of the placental sample, but not polymorphnuclear leukocyte. The blood samples of the patient were inoculated into aerobic and anaerobic blood culture bottles (bioMerieux, France). Aerobic and anaerobic blood cultures were evaluated by the BACTEC 9120 system (bioMerieux, France). While the aerobic cultures of the samples were negative, a Gram positive basil was recovered from Schaedler agar on which placental sample was inoculated and from the anaerobic blood culture bottle after a 48 hour incubation. The bacteria that demonstrated twice zone-hemolysis and had positive for spor-forming test were identified as Clostridium spp by classical methods. The identification was confirmed as C. perfringens $(99.9 \%$ confidence level) by the API 20A system (bioMerieux, France). Antibiotic susceptibility testing was performed by E-test (bioMerieux, France) according to recommendations of Clinical Laboratory and Standards Institute (CLSI) ${ }^{(2)}$. Resistance of the bacteria to penicillin was not found. The bacteria indicated positive reactions for glucose, lactose, saccarrose, maltose, mannose and trehalose fermentation, and hydrolysis of gelatin. Acid producing from mannitole, indol production, hydrolysis of urea, hydrolysis of esculin were negative.

\section{Conclusion}

The majority of cases of clostridial sepsis originate from the female genital tract and follow septic abortion. Introduction of a foreign body is a common antecedent event. In the uterus, residual necrotic fetal and placental tissues and traumatized endometrium may allow the growth of clostridia. There is often evidence of disseminated intravascular coagulation (DIC) ${ }^{(10)}$.

C.perfringens, the most commonly recovered Clostridium spp, is an inhabitant of soil and of intestines of humans and animals, and is the most frequently encountered histotoxic Clostridium species. This microorganism is easily isolated and identified in the clinical laboratory. It can be characterized by the presence of stout Gram- variable rods of varying length, frequently surrounded by a capsule. It's one of the anaerobic bacteria causing genital tract infections in female (8). Although Clostridia are isolated in up to $20 \%$ of non-STD genital infections, C.perfringens is rarely isolated from blood culture. According to the data of blood culture over a 5-year period from a large Boston teaching hospital, more than 20.000 positive blood cultures were detected with over 200 (1\%) positive for obligate anaerobes. Of the positive cultures, $58(0.3 \%)$ were positive for Clostridium spp. Of the Clostridium spp isolated, C. perfringens accounted for 42 isolates. Significant risk factors associated with isolation of clostridia from blood include hemodialysis, intestinal malignancy and inflammatory bowel disease ${ }^{(1)}$. Fujita et al, 18 C.perfringens bacteremias (0,13\%) among 14.190 blood samples were detected in a period of eight years in Japan ${ }^{(6)}$. In Turkey the rate of anaerobic bacteria isolated from blood cultures is approxiamately $1 \%$. Only one C.perfringens strain was isolated from blood cultures in our department in last year.

The further remarkable side of our case is that the patient had a diagnosis of fetal omphalocele (level 2). Omphalocele is an anterior abdominal wall defect in which abdominal organs protrude into the umbilical cord. The area of the defect is covered by a membrane consisting of the peritoneum, mesenchymal connective tissue and amnion. The diagnosis is commonly made during a routine fetal examination in the second trimester. Omphalocele is easily recognizable using ultrasound examination. Although the exact mechanism leading to omphalocele is controversial, the developmental failure can occur in the second or third trimester in embryonic or fetal life. Omphalocele affects 1 in 
4000 fetuses and has a birth prevalence of 1 in $6000^{(3,4)}$.

Cancer and immunosuppressive conditions are known to be the main underlying diseases in individuals infected with C. perfringens, but Fujita et al reported that the most frequent underlying conditions were diseases of the hepatobiliary tract system ${ }^{(6)}$. In this case, the patient whose blood culture C.perfringens was isolated from, had a diagnosis of fetal omphalocele.

The therapeutic regimens in genital tract infections in female include doxycycline or a macrolide in combination with cefoxitin, cefotetan, clindamycin or metronidazole ${ }^{(8)}$. Our patient started to take doxycycline and imipenem before culture results was available. She had continued on taking doxycycline according to blood and placental specimen culture results.

Intra-amniotic infection syndrome (IAIS), also referred to as chorioamnionitis, is a clinically detectable infection of the amniotic fluid and fetal membranes during pregnancy. Most cases of IAIS originate when vaginal microorganisms ascend into the intrauterine cavity after rupture of the membranes ${ }^{(11)}$. So it's considered that the cause of dissemination of C.perfringens to blood may be an ascendent transmission from maternal bowel flora, which then passes through the placenta and omphaloceled fetus. Due to the thickened abdominal layers, the fetus is increasingly sensitive to infections. Patients whose blood cultures were positive for $C$. perfringens had a mortality rate of $27 \%$ at 30 days ${ }^{(6)}$. Accurate microbiological diagnosis and early treatment provided to survival of the patient in this paper.

In conclusion, although Clostridium spp is rarely isolated from blood cultures, anaerobically evaluation of blood cultures in the infections of abdominal and genital sites could be life-saving.

\section{References}

[1] Onderdonk AB, Garrett WS. Gas gangrene and other Clostridium-associated diseses. Mandell, Douglas and Bennet's Principle and Practice of Infectious Diseases, $7^{\text {th }}$ edition (Eds: Mandell GL, Bennet JE, Dolin RD), Chapter 246, p.3103-9, Churchill Livingstone, Philadelphia, USA, 2010.
[2] Clinical and Laboratory Standards Institute (CLSI). Performance Standards for Antimicrobial Suscebtibility Testing. $21^{\text {st }}$ Informational Supplement. M100-S21, Vol 31, No 1, Table 2J (M02- M07: Anaerobes), Wayne, PA: Clinical and Laboratory Standards Institute, 2011.

[3] Kleinrouweler CE, Kuijper CF, Zalen-Sprock MM et al. Characteristics and outcome and the omphalocele circumference/abdominal circumference ratio in prenatally diagnosed fetal omphalocele. Fetal Diagn Ther 2011; 30: 60-69.

[4] Brantberg A, Blaas HGK, Haugens SE, Eik-Nes SH. Characteristics and outcome of 90 cases of fetal omphalocele. Ultrasound Obstet Gynecol 2005; 26: 527-537.

[5] Tassin M, Descriaud C, Elie C, Debarge VH, Dumez Y, Perrotin F, Benachi A. Omphalocele in the first trimester: prediction of perinatal outcome. Prenatal Diagnosis 2013; 33, 497-501

[6] Fujita H, Nishimura S, Kurosawa S, Akiya I, Nakamura-Uchiyama F, Ohnishi K. Clinical and Epidemiological Features of Clostridium perfringens Bacteremia: A Review of 18 Cases over 8 Year-Period in a Tertiary Care Center in Metropolitan Tokyo Area in Japan. Intern Med 2010; 49: 2433-2437

[7] Myers G, Ngoi SS, Cennerazzo W, Harris L, DeCosse JJ. Clostridial septicemia in an urban hospital. Surg Gynecol Obstet. 1992; 174 (4):291-6.

[8] Itzhak B. Anaerobic bacteria. "Cohen J, Powderly WG (eds):Infectious Diseases, Second edition, p.2265, Mosby, Elsevier,2004.

[9] https://www.luriechildrens.org/en-us/care-services/condition s-treatments/institute-fetal-health/Pages/basics/fetal-generalsurgery/fetal-omphalocele.aspx

[10] Kasper D, Madoff LC. Gas gangrene and other Clostridial infections. "Harrison's Infectious Diseases Kasper D, Fauci AS (eds), Chapter 42, p.438, McGraw-Hill Companies, USA, 2010 .

[11] Soper DE. Infections of the female pelvis. Mandell, Douglas and Bennet's Principle and Practice of Infectious Diseases, $7^{\text {th }}$ edition (Eds: Mandell GL, Bennet JE, Dolin RD), Chapter 108, p. 1511, Churchill Livingstone, Philadelphia, USA, 2010. 